Original article

\title{
Association between clusters of diseases and polypharmacy in hospitalized elderly patients: Results from the REPOSI study
}

\author{
Alessandro Nobili a,*, Alessandra Marengoni ${ }^{\text {b, }}{ }^{\text {, Mauro Tettamanti }}{ }^{\text {, }}$, Francesco Salerno ${ }^{\mathrm{c}, 1}$, Luca Pasina ${ }^{\mathrm{a}}$, \\ Carlotta Franchi ${ }^{\text {a }}$, Alfonso Iorio ${ }^{\mathrm{d}, 1}$, Maura Marcucci ${ }^{\mathrm{e}}$, Salvatore Corrao ${ }^{\mathrm{f}}$, \\ Giuseppe Licata $^{\mathrm{f}, 1}$, Pier Mannuccio Mannucci ${ }^{\mathrm{g}, 1}$ \\ a "Mario Negri" Institute for Pharmacological Research, Milano, Italy \\ b Department of Medical and Surgery Sciences, University of Brescia, Geriatric Ward, Spedali Civili, Brescia, Italy \\ ${ }^{c}$ Medicina Interna, IRCCS Policlinico San Donato, University of Milano, Italy \\ d Clinical Epidemiology E' Biostatistics, McMaster University, Hamilton, Canada \\ e Department of Internal Medicine, University of Perugia, Italy \\ f Dipartimento Biomedico di Medicina Interna e Specialistica, University of Palermo, Italy \\ g Scientific Direction, IRCCS Cà Granda Foundation Maggiore Policlinico Hospital, Milan, Italy
}

\section{A R T I C L E I N F O}

\section{Article history:}

Received 1 June 2011

Received in revised form 12 July 2011

Accepted 26 August 2011

Available online 29 September 2011

\section{Keywords:}

Polypharmacy

Cluster of diseases

Hospitalized elderly

\begin{abstract}
A B S T R A C T
Background: Although the association between multimorbidity and polypharmacy has been clearly documented, no study has analyzed whether or not specific combinations of diseases influence the prescription of polypharmacy in older persons. We assessed which clusters of diseases are associated with polypharmacy in acute-care elderly inpatients.

Methods: This cross-sectional study was held in 38 Italian internal medicine and geriatric wards participating in the Registro Politerapie SIMI (REPOSI) study during 2008. The study sample included 1155 in-patients aged 65 years or older. Clusters of diseases, defined as two or more co-occurring specific chronic diseases, were identified using the odds ratio (OR) for the associations between pairs of diseases followed by cluster analysis. Polypharmacy was defined as the prescription of five or more different medications at hospital discharge. Logistic regression models were run to analyze the association between clusters of diseases and polypharmacy.

Results: Among clusters of diseases, the highest mean number of drugs $(>8)$ was found in patients affected by heart failure (HF) plus chronic obstructive pulmonary disease (COPD), HF plus chronic renal failure (CRF), COPD plus coronary heart disease (CHD), diabetes mellitus plus CRF, and diabetes mellitus plus CHD plus cerebrovascular disease (CVD). The strongest association between clusters of diseases and polypharmacy was found for diabetes mellitus plus CHD plus CVD, diabetes plus CHD, and HF plus atrial fibrillation (AF).

Conclusions: The observed knowledge of the relationship among co-occurring diseases and polypharmacy should help to identify and monitor older in-patients at risk of polypharmacy.
\end{abstract}

(c) 2011 European Federation of Internal Medicine. Published by Elsevier B.V. All rights reserved.

\section{Introduction}

Polypharmacy is very common in older adults and may well be needed to improve symptoms, disease-related problems and quality of life [1-3]. Although polypharmacy has been defined in different ways, we consider it as the use of five or more medications regardless of whether they are necessary or unnecessary. Polypharmacy may also be a major risk for inappropriate prescribing, poor adherence to therapies, adverse drug events and other adverse health outcomes [4-7]. Prevalence and

\footnotetext{
* Corresponding author at: Laboratory for Quality Assessment of Geriatric Therapies and Services, Mario Negri Institute for Pharmacological Research, via Giuseppe La Masa, 19, 20156 Milan, Italy. Tel.: + 3902 39014512; fax: + 390239001916.

E-mail address: alessandro.nobili@marionegri.it (A. Nobili).

${ }^{1}$ On behalf of SIMI Investigators (SIMI, Italian Society of Internal Medicine. The participating hospitals and co-authors are listed in Appendix A.).
}

predictors of polypharmacy have been analyzed in many studies, with results differing in relation to the clinical setting $[3,8,9]$, but usually the prevalence of polypharmacy is closely related to the number of diseases a person has (i.e. multimorbidity) [3,5]. The prevalence of multimorbidity in older persons ranges from $35 \%$ to $80 \%$, depending on the data collection method, the definitions of chronic conditions and multimorbidity, and the number of chronic conditions included in the analysis [10-15], but is likely to rise in coming years with the aging of the population [14]. Furthermore, elderly people with multimorbidity are at high risk of hospitalization because of acute events or complications of chronic conditions, and hospitalization itself is one of the most important risk factors for polypharmacy $[16,17]$.

Besides multimorbidity, specific chronic diseases such as hypertension, coronary heart disease (CHD), heart failure (HF), chronic obstructive pulmonary disease (COPD), chronic renal failure (CRF) and diabetes mellitus are predictors of polypharmacy $[13,14,18-20]$. However, to our 
knowledge no study has analyzed whether or not the clustering of specific chronic diseases influences polypharmacy in elderly in-patients. Since diseases often cluster together beyond what would be expected by chance [14,15,21], knowledge of how diseases co-occur might help clinicians shift from a disease-based perspective to a patient-based perspective when prescribing drugs.

With this as background, the aim of this study was to assess which clusters of diseases were associated with polypharmacy at hospital discharge in Italian elderly persons in acute care wards.

\section{Material and methods}

\subsection{Data collection}

The study was conducted between January 2008 and December 2008 in 38 hospitals in different regions of Italy, all participating in the Registro Politerapie SIMI (REPOSI) study, organized in collaboration by the Italian Society of Internal Medicine (SIMI) and the Mario Negri Institute for Pharmacological Research. The REPOSI study was designed to set up a network of internal medicine and geriatric wards to evaluate patients with multiple diseases and multiple drugs. Participation in the network was voluntary, but attention was paid in the choice of the participating centers to their homogeneous composition in terms of geographic distribution, size and unselected admissions from the territory or the emergency room.

The specific aims of the REPOSI study were: to describe the prevalence of concurrent multiple diseases and treatments in elderly in-patients; to correlate their clinical characteristics with the type and number of diseases and treatments and to examine the main clinical outcomes at hospital discharge. The study had two phases, the first to create the network of internal medicine and geriatric wards, and the second to set up a registry of patients included in the study.

All patients 65 years old or older admitted to the wards participating in the study were consecutively recruited. Participation was voluntary and all patients signed informed consent. Data collection complied fully with Italian law on personal data protection. Under the applicable legal principles on patients' registries, the study did not require ethical committee approval. At least 40 patients consecutively admitted to each participating center during four separate weeks, about three months apart (in February, June, September, and December 2008) were included in the study. A standardized web-based Case Report Form was filled in by the attending physicians, including socio-demographic factors, clinical variables, diagnoses and treatments at hospital admission and discharge, clinical events during hospitalization and outcome. All the data entered were collected and checked for quality and consistency by a central monitor of the coordinating center (Mario Negri Institute for Pharmacological Research, Milan).

During each index week, all wards had to complete the register of all patients admitted to the ward, and indicate those who were consecutively enrolled in the study. For patients who were excluded, the reason had to be given. On the basis of these data, during the four weeks, the recruitment rate for each ward was nearly $40 \%$ of patients admitted. Sixty eight percent of those excluded were because of age under 65 years. Other reasons for exclusion were refusal to participate or to sign informed consent (23\%), seriousness of patient's clinical condition or admission in terminal state (6\%), and other reasons (3\%). No difference for age and sex (the only available data) emerged for these patients in comparison to the enrolled sample.

The initial study sample included 1411 patients; 79 (5.6\%) were excluded because of missing or incomplete data, 25 for missing data on hospital outcome and 54 for errors in data input and recording of socio-demographic and clinical characteristics. Thus, 1332 were available for the overall analyses. Patients who were not discharged to home $(n=111)$ were excluded. Of these, 6 were terminally ill at hospital admission and were transferred to end-of-life care structures; 44 were transferred to rehabilitation units or long-term facilities, and 61 to other hospital units for acute medical or surgical conditions arising in hospital. In addition, 66 patients died during the hospital stay leaving 1155 individuals available for the final analyses.

\subsection{Assessment of diseases}

Diseases examined in this study were collected at hospital admission and confirmed by clinical examination, clinical history and laboratory and instrumental data collected by the attending physicians. Diagnoses were made using standardized criteria. The International Classification of Diseases - Ninth Revision (ICD-9) (WHO) (http:// icd9cm.chrisendres.com/ accessed January 18,2010 ) was used for classifying all the diseases (Table 1a). Only diseases with prevalence of $>5 \%$ $(\mathrm{n}=19)$ were taken into account.

For the purpose of this study, clusters of diseases were defined as two or more co-occurring specific conditions, according to Boyd and colleagues [20]. Diseases' clusters were identified in a previous study held in the same population using logistic regression models to analyze the bivariate associations between pairs of diseases and cluster analysis (Table 1b) [15,21]. The Charlson Index was employed to examine the coexistence and severity of multiple diseases [22]. Each condition is assigned a score of $1,2,3$ or 6 depending on the associated risk of dying. The scores are summed up and the total predicts mortality [22].

\subsection{Drug prescription and polypharmacy}

Drugs were recorded according to the Anatomical Therapeutic Chemical classification system (ATC) (WHO) (http://www.whocc.no/ atc_ddd_index/ accessed January 18, 2010.). Although there is still no consensus or commonly used cut-off for polypharmacy, we set the cut-off at five or more different drugs, because this was the median number of drugs per patient at hospital admission in the enrolled patients, and because it is one of the most frequently used cut-offs in the literature on polypharmacy $[2,3,23]$. Moreover, this cut-off was the same used in another published study by REPOSI group [24].

When we computed the presence of polypharmacy, pills containing a fixed association of two active substances were counted as only one drug.

\subsection{Adverse clinical events}

Adverse clinical events were defined as any new acute clinical problem that arose in hospital [25].

Table 1a

ICD-9 codes used to classifying diseases (corresponding diseases are listed in alphabetical order).

\begin{tabular}{ll}
\hline Diagnoses & ICD-9 codes \\
\hline Anemia & $280-285$ \\
Anxiety & 300 \\
Arthritis & 715 \\
Atrial fibrillation (AF) & 427 \\
Cerebrovascular diseases (CVD) & $430-438$ \\
Coronary heart disease (CHD) & $410-414$ \\
Chronic obstructive pulmonary disease (COPD) & $490-496$ \\
Chronic renal failure (CRF) & 585 \\
Dementia & 290,331 \\
Diabetes mellitus & 250 \\
Dyslipidemia & 272 \\
Gastric diseases & $530-536$ \\
Heart failure (HF) & 428 \\
Hypertension & $401-405$ \\
Intestinal diseases & $560-569$ \\
Liver cirrhosis & 571 \\
Malignancy & $140-165,170-175,179-208$ \\
Prostate hypertrophy & 600 \\
Thyroid diseases & $240-246$ \\
\hline
\end{tabular}


Table 1b

Clusters of diseases previously identified [21] listed in alphabetical order.

\begin{tabular}{l}
\hline Clusters \\
\hline COPD and CHD \\
CRF and anemia \\
Dementia and arthritis \\
Diabetes and CHD \\
Diabetes and CRF \\
Diabetes and CVD \\
Diabetes and CHD and CVD \\
Diabetes and dyslipidemia \\
Gastric and intestinal diseases \\
HF and AF \\
HF and COPD \\
HF and CRF \\
Hypertension and CVD \\
Hypertension and diabetes \\
Hypertension and dyslipidemia \\
Liver cirrhosis and anemia \\
Liver cirrhosis and malignancy \\
Thyroid dysfunction and AF \\
\hline
\end{tabular}

CHD $=$ Coronary Heart Disease, $\mathrm{AF}=$ Atrial Fibrillation, COPD = Chronic Obstructive Pulmonary Disease, $\mathrm{CVD}=$ Cerebrovascular Disease, $\mathrm{CRF}=$ Chronic Renal Failure, $\mathrm{HF}=$ Heart Failure.

\subsection{Statistical analysis}

Several logistic regression models were run to analyze the associations between each disease and cluster of diseases with polypharmacy. The adjustment included age (years), sex, education (years of schooling), the Charlson Index (score) and participating centers. Further adjustment for length of hospital stay and adverse clinical events in hospital failed to significantly change the results and to improve the model. All statistical calculations were done with STATA software 9th version (College Station, Texas, US).

\section{Results}

Of the 1155 patients in the analyses, 54\% was women. The mean age of the patients was 79.2 years (95\% CI: 78.4-80.1) and the mean hospital stay was 11 days (95\% CI: 10.2-12.0). The most frequent diagnoses at admission were: hypertension followed by diabetes mellitus, CHD, AF, CVD, and COPD, and their prevalence was significantly higher in patients prescribed with polypharmacy (Table 2). The average number of drugs prescribed at hospital discharge was 5.9 (95\% CI: 5.6-6.3); 774 (67\%) patients were discharged with polypharmacy. One third had at least one adverse clinical event during the hospital stay (range 0-9) (Table 2). The most frequent were urinary infection $(12.0 \%)$, fever $(6.0 \%)$, anemia $(5.2 \%)$, pneumonia $(5.0 \%)$, electrolyte disorders (4.5\%), atrial fibrillation (4.3\%), heart failure (3.0\%), and acute renal failure $(2.7 \%)$.

The mean number of prescribed drugs was the highest $(>7)$ in patients with diabetes mellitus, CHD, COPD, CRF and HF, and the lowest $(<6)$ in patients with dementia (Table 3$)$. In logistic regression models several diseases emerged as significantly associated with the prescription of polypharmacy at hospital discharge. The association was strongest for cardiovascular diseases: CHD (OR 4.0; 95\% CI: 2.7-6.1), HF (OR 3.6; 95\% CI: 1.6-8.1), and AF (OR 2.7; 95\% CI: 1.9-3.7). Malignancy, gastric diseases, anemia, anxiety, prostate hypertrophy, liver cirrhosis, dementia, arthritis and intestinal diseases were not independently associated with polypharmacy (Table 3).

Finally, Table 4 shows the mean number of prescribed drugs according to disease clusters and the results of further logistic regression models testing the association between different disease clusters and polypharmacy. The mean number of prescribed drugs was the highest $(>8)$ in patients with HF plus COPD, HF plus CRF, COPD plus CHD, diabetes mellitus plus CRF, and diabetes mellitus plus CHD plus CVD, and the
Table 2

Socio-demographic characteristics of the patients and prevalence of diseases in the whole sample and according to polypharmacy. Data are given as means or proportions (95\% confidence intervals) adjusted for participating centers.

\begin{tabular}{lccc}
\hline & $\begin{array}{l}\text { All } \\
(\mathrm{n}=1155)\end{array}$ & $\begin{array}{l}5+\text { drugs } \\
(\mathrm{n}=774)\end{array}$ & $\begin{array}{l}<\text { drugs } \\
(\mathrm{n}=381)\end{array}$ \\
\hline Age, yrs, mean & $79.2(78.4-80.1)$ & $78.9(77.9-79.8)$ & $79.8(78.8-80.8)$ \\
Women & $53.6(49.4-57.9)$ & $53.5(48.4-58.6)$ & $53.9(48.6-59.3)$ \\
Education, yrs, mean & $6.3(5.7-6.9)$ & $6.5(5.9-7.2)$ & $5.9(5.2-6.6)$ \\
Number of drugs, mean & $5.9(5.6-6.3)$ & $7.5(7.2-7.7)$ & $3.0(2.9-3.1)$ \\
Number of adverse & $0.6(0.3-0.8)$ & $0.6(0.4-0.9)$ & $0.5(0.2-0.8)$ \\
$\quad$ clinical events, mean & & & \\
At least one adverse & $33.2(25.8-40.7)$ & $35.1(27.1-43.1)$ & $29.5(20.5-38.4)$ \\
$\quad$ clinical event & & & \\
Charlson Index, mean & $2.9(2.7-3.2)$ & $3.3(3.1-3.6)$ & $2.2(1.8-2.6)$ \\
Hospital stay, days, mean & $11.1(10.2-12.0)$ & $11.5(10.5-12.5)$ & $10.5(9.1-11.8)$ \\
Hypertension & $58.8(52.8-64.9)$ & $65.2(59.1-71.2)$ & $46.1(39.9-53.1)$ \\
Diabetes mellitus & $24.3(20.7-27.9)$ & $29.4(24.9-33.9)$ & $13.9(10.6-17.3)$ \\
CHD & $22.9(18.8-27.0)$ & $29.7(24.6-34.7)$ & $9.2(5.9-12.5)$ \\
AF & $20.0(16.8-23.3)$ & $24.4(20.3-28.4)$ & $11.3(8.3-14.4)$ \\
COPD & $19.3(16.2-22.2)$ & $23.1(19.2-26.9)$ & $11.6(7.9-15.3)$ \\
CVD & $20.1(15.7-24.4)$ & $22.7(17.6-27.7)$ & $14.7(10.9-18.5)$ \\
Malignancy & $14.7(11.2-18.1)$ & $14.8(11.2-18.4)$ & $14.5(9.1-19.9)$ \\
Gastric diseases & $14.2(9.4-19.0)$ & $16.1(10.2-21.9)$ & $10.1(6.1-14.9)$ \\
Dyslipidemia & $13.9(9.9-18.0)$ & $16.8(12.1-21.5)$ & $8.2(4.7-11.6)$ \\
CRF & $11.5(8.8-14.3)$ & $14.6(11.3-18.0)$ & $5.3(3.1-7.4)$ \\
Anemia & $9.1(6.6-11.6)$ & $9.3(6.6-12.1)$ & $8.7(5.5-11.8)$ \\
Thyroid diseases & $9.4(6.9-11.8)$ & $11.5(8.6-14.5)$ & $5.0(2.6-7.4)$ \\
HF & $8.2(5.4-11.1)$ & $10.8(7.2-14.4)$ & $3.2(5.2-5.8)$ \\
Anxiety & $7.9(5.7-10.1)$ & $9.1(6.4-11.7)$ & $5.5(2.8-8.3)$ \\
Prostate hypertrophy & $7.8(5.6-10.1)$ & $8.9(6.4-11.4)$ & $5.5(2.5-8.6)$ \\
Liver cirrhosis & $8.2(5.3-11.0)$ & $8.5(5.3-11.8)$ & $7.4(3.6-11.1)$ \\
Dementia & $6.9(4.2-9.7)$ & $6.5(3.5-9.4)$ & $7.9(4.3-11.5)$ \\
Arthritis & $6.9(4.6-9.3)$ & $7.3(4.5-10.0)$ & $6.3(3.1-9.5)$ \\
Intestinal diseases & $7.5(5.2-9.7)$ & $8.4(6.1-10.7)$ & $5.5(2.6-8.5)$ \\
\hline
\end{tabular}

$\mathrm{CHD}=$ Coronary Heart Disease, $\mathrm{AF}=$ Atrial Fibrillation, $\mathrm{COPD}=$ Chronic Obstructive Pulmonary Disease, $\mathrm{CVD}=$ Cerebrovascular Disease, $\mathrm{CRF}=$ Chronic Renal Failure, $\mathrm{HF}=$ Heart Failure.

lowest $(<6)$ in patients with dementia plus arthritis. The association between clusters of diseases and polypharmacy was strongest for diabetes mellitus plus CHD plus CVD (OR 9.8; 95\% CI: 1.3-72.2).

Table 3

Number of patients affected by specific diseases (N), mean number of drugs (95\% confidence intervals, $\mathrm{CI}$ ) and odds ratios (OR) for polypharmacy according to single diseases. Findings adjusted for age, sex, education, Charlson Index and participating centers.

\begin{tabular}{lrlll}
\hline Diseases & \multicolumn{1}{c}{$\mathrm{N}$} & Mean no. of drugs $(95 \% \mathrm{CI})$ & OR & 95\% CI \\
\hline CHD & 264 & $7.5(6.9-7.9)$ & 4.0 & $2.7-6.1$ \\
HF & 95 & $7.3(6.5-8.2)$ & 3.6 & $1.6-8.1$ \\
AF & 234 & $6.7(6.2-7.1)$ & 2.7 & $1.9-3.7$ \\
Dyslipidemia & 161 & $6.7(6.1-7.3)$ & 2.4 & $1.6-3.7$ \\
Thyroid diseases & 108 & $6.8(6.2-7.4)$ & 2.4 & $1.4-4.1$ \\
Hypertension & 679 & $6.4(6.0-6.8)$ & 2.3 & $1.8-2.9$ \\
CRF & 135 & $7.5(6.9-8.2)$ & 2.1 & $1.3-3.3$ \\
Diabetes mellitus & 281 & $7.4(6.9-7.8)$ & 1.9 & $1.4-2.8$ \\
COPD & 223 & $7.2(6.4-7.9)$ & 1.9 & $1.3-2.9$ \\
Prostate hypertrophy & 90 & $6.8(6.1-7.6)$ & 1.9 & $0.9-3.6$ \\
Intestinal diseases & 87 & $6.4(5.9-6.9)$ & 1.8 & $0.9-3.3$ \\
Gastric diseases & 164 & $6.7(6.1-7.2)$ & 1.6 & $0.9-2.6$ \\
Anxiety & 91 & $6.5(5.6-7.5)$ & 1.6 & $0.9-2.7$ \\
CVD & 231 & $6.4(5.9-6.9)$ & 1.5 & $1.1-2.0$ \\
Arthritis & 80 & $6.1(5.3-6.8)$ & 1.2 & $0.7-2.1$ \\
Anemia & 107 & $6.4(5.7-7.1)$ & 0.9 & $0.6-1.3$ \\
Liver cirrhosis & 96 & $6.2(5.5-6.8)$ & 0.8 & $0.4-1.4$ \\
Dementia & 80 & $5.3(4.6-6.0)$ & 0.8 & $0.5-1.3$ \\
Malignancy & 170 & $6.2(5.5-6.8)$ & 0.6 & $0.4-0.9$ \\
\hline
\end{tabular}

$\mathrm{CVD}=$ Cerebrovascular Disease HF $=$ Heart Failure, $\mathrm{AF}=$ Atrial Fibrillation, $\mathrm{COPD}=$ Chronic Obstructive Pulmonary Disease, $\mathrm{CRF}=$ Chronic Renal Failure, $\mathrm{CHD}=$ Coronary Heart Disease. 
Table 4

Mean number of drugs (95\% confidence intervals, $\mathrm{CI}$ ) and odds ratios (OR) for polypharmacy according to clusters of diseases. Findings adjusted for age, sex, education, Charlson Index and participating centers.

\begin{tabular}{llll}
\hline Clusters of diseases & Mean no. of drugs (95\% CI) & OR & 95\% CI \\
\hline Diabetes and CHD and CVD & $8.3(7.1-9.5)$ & 9.8 & $1.3-72.2$ \\
Diabetes and CHD & $8.7(7.9-9.5)$ & 5.8 & $2.6-13.2$ \\
HF and AF & $7.6(6.7-8.5)$ & 5.5 & $2.0-14.9$ \\
Thyroid dysfunction and AF & $7.4(6.7-8.1)$ & 5.0 & $1.8-14.1$ \\
COPD and CHD & $8.3(7.3-9.2)$ & 4.1 & $1.7-9.9$ \\
Hypertension and dyslipidemia & $7.1(6.5-7.7)$ & 3.7 & $2.0-6.7$ \\
HF and COPD & $8.7(7.4-9.9)$ & 3.7 & $0.8-16.8$ \\
Diabetes and CVD & $7.4(6.7-8.0)$ & 3.2 & $1.5-6.7$ \\
Diabetes and dyslipidemia & $7.9(7.2-8.6)$ & 2.8 & $1.3-6.1$ \\
Diabetes and CRF & $8.3(7.4-9.2)$ & 2.7 & $0.9-7.9$ \\
Hypertension and diabetes & $7.7(7.1-8.2)$ & 2.4 & $1.6-3.5$ \\
Hypertension and CVD & $6.7(6.3-7.1)$ & 2.3 & $1.7-3.0$ \\
HF and CRF & $8.2(6.8-9.6)$ & 2.2 & $0.4-11.5$ \\
CRF and anemia & $7.9(6.5-9.3)$ & 1.8 & $0.7-4.8$ \\
Gastric and gastro-intestinal diseases & $7.1(6.0-8.1)$ & 1.8 & $0.6-5.4$ \\
Arthritis and dementia & $5.7(3.8-7.5)$ & 0.9 & $0.2-4.5$ \\
Liver cirrhosis and anemia & $6.5(4.9-8.1)$ & 0.7 & $0.2-3.0$ \\
Liver cirrhosis and malignancy & $6.1(4.9-7.3)$ & 0.6 & $0.3-1.6$ \\
\hline
\end{tabular}

$\mathrm{CVD}=$ Cerebrovascular Disease; $\mathrm{HF}=$ Heart Failure, $\mathrm{AF}=$ Atrial Fibrillation, $\mathrm{COPD}=$ Chronic Obstructive Pulmonary Disease, $\mathrm{CRF}=$ Chronic Renal Failure, $\mathrm{CHD}=$ Coronary Heart Disease.

\section{Discussion}

This study of hospitalized elderly Italian patients examined the association between the co-occurrence of specific chronic diseases and polypharmacy by a cluster approach [15,21]. Diseases often cluster together beyond what would be expected by chance, raising the question of a possible additive or multiplicative effect on different health-related outcomes. As reported in a previous study on the same population, patients with different clusters of diseases not only had different mortality rate and clinical adverse events in hospital, but specific combinations of diseases had additive effect on these health outcomes [21]. In the present study, the mean number of drugs ranged from 5.7 for the cluster arthritis plus dementia to 8.7 for the clusters HF plus COPD and diabetes plus CHD. Polypharmacy was strongly associated with the clusters of diabetes plus CHD and CVD, diabetes plus CHD, HF plus AF, and thyroid dysfunction plus AF, after adjustment for demographic characteristics, the Charlson Index and participating centers. The co-occurrence of diseases had additive effects on the risk of polypharmacy for diabetes with CHD and CVD, diabetes plus CHD, AF plus HF, CVD plus diabetes, COPD plus $\mathrm{CHD}$, and thyroid dysfunction plus AF.

Among the main findings of this study is that diabetes mellitus, one of the most frequent diseases of the elderly, tends to cluster; in fact it was found in six clusters. Considered as a single disease, diabetes was associated with nearly double the risk of polypharmacy, but clustered with other conditions, especially cardiovascular diseases, showed a much greater increase in the prescription of polypharmacy compared to diabetes alone, even after multi-adjustment. Thus, the risk of polypharmacy in the elderly patients with diabetes mellitus might be explained by the high probability of co-occurring diseases. In diabetics polypharmacy is often unavoidable, since multiple drug therapy has become the standard for most of its common comorbidities, and the potential for marked polypharmacy is likely continue to rise as more therapeutic options become available [19,26].

Besides diabetes, cardiovascular diseases also influence the clustering. These were the most common pathologies with an overall prevalence ranging from nearly $60 \%$ for hypertension to $20 \%$ for $\mathrm{AF}$ and CVD. The co-occurrence of HF and AF and HF and COPD increased the risk of polypharmacy in comparison with the single diseases in the clusters. Extensive use of multiple drugs is common in these patients and is recommended by international guidelines [27,28]. Advances in cardiovascular drug treatment have increased life-expectancy. However, polypharmacy is neither always efficacious nor safe, and often leads to inappropriate drug use, poor compliance, increased morbidity and costs [18]. In older persons, the risks of polypharmacy and the potential for inappropriate therapy must be considered and balanced against the possible benefits of multiple drug therapies. There is no agreement in the literature about the benefits of treating older patients with multiple drugs, especially if they are prescribed for primary or secondary prevention. One of the reasons is that old patients and particularly those very old or affected by multimorbidity are rarely included in studies on treatments' outcomes. A new approach to reducing the risks and maximizing the benefits of polypharmacy might include the identification of patient groups particularly at risk of being prescribed with polypharmacy. The disease clustering approach, when evaluating older patients, may help in monitoring these patients. Furthermore, the next step in this field should be the identification of the optimal treatment, in term of both number of medications and most important appropriateness of prescription, for each cluster of diseases, especially for high frequency clusters, or clusters particularly difficult to be treated.

In the present study polypharmacy was not significantly associated with clusters of chronic disease such as liver cirrhosis and anemia, liver cirrhosis and malignancy, CRF and anemia, conditions which largely mark the terminal phases of life. In the last years of life the number of prescribed drugs can drop compared to younger ages [1,5,9,29]. We found similar results for dementia alone or clustered with other conditions. One possible explanation might be that patients with dementia may be undertreated compared with non-demented ones [30]. Another explanation may be that the severity of dementia may have limited the applicability of the possible therapeutic options for these patients. Unfortunately, data on the severity of dementia and other diseases were not available in this study.

\subsection{Strengths and limitations}

The major strengths of the REPOSI study include the multicenter design that involved 38 internal medicine and geriatric wards throughout Italy, resulting in a sample representative of the hospitalized elderly population; second, patients were enrolled in four different weeks (one per season) in order to balance the effect of seasons on the acute diseases leading to hospital admission. However, a few limitations must also be mentioned. First, the severity of diseases was not taken into account, in consideration that the evaluation of disease severity is usually made difficult owing to several classification scales or requirement of subjective judgments. Another limitation is the lack of information on appropriateness of drug prescribed. However, although the absolute number of drugs cannot be considered a direct indicator of prescribing appropriateness $[2,5,20]$, there is growing evidence that polypharmacy is associated with increases in many adverse outcomes, including adverse drug reactions, drug-drug or drug-disease interactions, falls, hospital admission, and mortality [4,5,7,9,23]. A further weakness is that problems can arise when using hospital data for research, because hospital records are not designed for this purpose but rather for patient care, and their diagnostic quality may vary depending on hospitals, physicians and clinical units. Moreover, admissions are often selective on the basis of local characteristics, associated medical conditions and admission policies, which can vary from hospital to hospital. Finally, the REPOSI data set was not planned to include a multidimensional geriatric assessment, because it is not general practice in internal medicine wards in Italy. Thus we have no information on patients' functional profiles.

\section{Learning points}

- Although the association between multimorbidity and polypharmacy has been clearly documented, no study has analyzed whether or not specific combinations of diseases influence the prescription of polypharmacy in older persons. 
- Polypharmacy is strongly associated with the clusters of diabetes plus CHD and CVD, diabetes plus CHD, HF plus AF, and thyroid dysfunction plus AF, after adjustment for demographic characteristics, the Charlson Index and participating centers.

- The co-occurrence of these diseases had additive effects on the risk of polypharmacy.

- When evaluating older patients, the disease clustering approach might help clinicians to identify patients particularly at risk of being prescribed with polypharmacy.

\section{Conflict of interest}

All the authors declare that no conflict of interest exists.

\section{Financial disclosure}

Carlotta Franchi holds a fellowship granted by Rotary Clubs Milano Naviglio Grande San Carlo, Milano Scala and Inner Wheel Milano San Carlo.

\section{Sponsor role}

None: the REPOSI study is a network of Italian internal medicine hospital wards which on a voluntary basis, and without any financial support, agreed to participate in data collection during the four index weeks.

\section{Funding sources}

Nothing.

\section{Acknowledgments}

We thank Professor Farncesco Violi, President of the Italian Society of Internal Medicine, for his help and encouragement. We are grateful to Judith Baggott for editorial assistance.

\section{Appendix A. REPOSI collaborators and participating units}

The following hospital and investigators have contributed to this study: Pier Mannuccio Mannucci, Alberto Tedeschi, Raffaella Rossio (Medicina Interna 2, Fondazione IRCCS Ospedale Maggiore, Milano); Guido Moreo, Barbara Ferrari (Medicina Interna 3, Fondazione IRCCS Ospedale Maggiore, Milano); Cesare Masala, Antonio Mammarella, Valeria Raparelli (Medicina Interna, Università La Sapienza, Roma); Nicola Carulli, Stefania Rondinella, Iolanda Giannico (Medicina Metabolica, Università di Modena e Reggio Emilia); Leonardo Rasciti, Silvia Gualandi (Medicina Interna, Policlinico S. Orsola Malpighi, Bologna); Valter Monzani, Valeria Savojardo (Medicina d'Urgenza, IRCCS Fondazione Ospedale Maggiore, Milano); Maria Domenica Cappellini, Giovanna Fabio, Flavio Cantoni (Medicina Interna 1A, Fondazione IRCCS Ospedale Maggiore, Milano); Franco Dallegri, Luciano Ottonello, Alessandra Quercioli, Alessandra Barreca (Medicina Interna 1, Università di Genova); Riccardo Utili, Emanuele Durante-Mangoni, Daniela Pinto (Medicina Interna, Seconda Università di Napoli); Roberto Manfredini, Elena Incasa, Emanuela Rizzioli (Medicina Interna, Azienda USL, Ferrara); Massimo Vanoli, Gianluca Casella (Medicina Interna, Ospedale di Lecco, Merate); Giuseppe Musca, Olga Cuccurullo (Medicina Interna, P.O. Cetraro, ASP Cosenza); Laura Gasbarrone, Giuseppe Famularo, Maria Rosaria Sajeva (Medicina Interna, Ospedale San Camillo Forlanini, Roma); Antonio Picardi, Dritan Hila (Medicina Clinica-Epatologia, Università Campus Bio-Medico, Roma); Renzo Rozzini, Alessandro Giordano (Fondazione Poliambulanza, Brescia); Andrea Sacco, Antonio Bonelli, Gaetano Dentamaro (Medicina, Ospedale Madonna delle Grazie, Matera); Francesco Salerno, Valentina Monti, Massimo Cazzaniga (Medicina Interna, IRCCS Policlinico San Donato, Università di Milano); Ingrid Nielsen, Piergiorgio
Gaudenzi, Lisa Giusto (Medicina ad Alta Rotazione, Azienda Ospedaliera Universitaria, Ferrara); Enrico Agabiti Rosei, Damiano Rizzoni, Luana Castoldi (Clinica Medica, Università di Brescia); Daniela Mari, Giuliana Micale (Medicina Generale ad indirizzo Geriatrico, IRCCS Istituto Auxologico Italiano, Milano); Emanuele Altomare, Gaetano Serviddio, Santina Salvatore (Medicina Interna, Università di Foggia); Carlo Longhini, Cristian Molino (Clinica Medica, Azienda Mista Ospedaliera Universitaria Sant'Anna, Ferrara); Giuseppe Delitalia, Silvia Deidda, Luciana Maria Cuccuru (Clinica Medica, Azienda Mista Ospedaliera Universitaria, Sassari); Giampiero Benetti, Michela Quagliolo, Giuseppe Riccardo Centenaro (Medicina 1, Ospedale di Melegnano, Vizzolo Predabissi, Milano); Alberto Auteri, Anna Laura Pasqui, Luca Puccetti (Medicina Interna, Azienda Ospedaliera Universitaria Le Scotte, Siena); Carlo Balduini, Giampiera Bertolino, Piergiorgio Cavallo (Dipartimento di Medicina Interna, Fondazione IRCCS Policlinico San Matteo, Università degli Studi di Pavia); Esio Ronchi, Daniele Bertolini, Nicola Lucio Liberato (Medicina Interna, Ospedale Carlo Mira, Casorate Primo, Pavia); Antonio Perciccante, Alessia Coralli (Medicina, Ospedale San GiovanniDecollato-Andisilla, Civita Castellana); Luigi Anastasio, Leonardo Bertucci (Medicina Generale, Ospedale Civile Serra San Bruno); Giancarlo Agnelli, Ana Macura, Alfonso Iorio, Maura Marcucci (Medicina Interna e Cardiovascolare, Ospedale Santa Maria della Misericordia, Università di Perugia); Cosimo Morabito, Roberto Fava (Medicina, Ospedale Scillesi d'America, Scilla); Giuseppe Licata, Antonino Tuttolomondo, Riccardo Di Sciacca (Medicina Interna e Cardioangiologia, Università degli Studi di Palermo); Luisa Macchini, Anna Realdi (Clinica Medica 4, Università di Padova); Luigi Cricco, Alessandra Fiorentini, Cristina Tofi (Geriatria, Ospedale di Montefiascone); Carlo Cagnoni, Antonio Manucra (UO Medicina e Primo Soccorso, Ospedale di Bobbio, Azienda USL di Piacenza);Giuseppe Romanelli, Alessandra Marengoni, Francesca Bonometti (UO Geriatria, Spedali Civili di Brescia); Michele Cortellaro, Maria Rachele Meroni, Marina Magenta (Medicina 3, Ospedale Luigi Sacco, Università di Milano); Carlo Vergani, Dionigi Paolo Rossi (Geriatria, Fondazione IRCCS Ospedale Maggiore e Università di Milano).

Clincal data monitoring and revision: Valentina Spirito, Damia Noce, Jacopo Bonazzi, Rossana Lombardo, Eleonora Sparacio, Luigi De Vittorio (Istituto di Ricerche Farmacologiche "Mario Negri”, Milano).

\section{References}

[1] Veehof LJG, Stewart RE, Haaijer-Raskamp FM, Meyboom-de Jong B. The development of polypharmacy. A longitudinal study. Fam Pract 2000;17:261-7.

[2] Gurwitz JH. Polypharmacy. A new paradigm for quality drug therapy in the elderly? Arch Intern Med 2004;164:1957-9.

[3] Hajjar ER, Cafiero AC, Hanlon JT. Polypharmacy in elderly patients. Am J Geriatr Pharmacother 2007:5:345-51.

[4] Hilmer SN, Gnjidic D. The effects of polypharmacy in older adults. Clin Pharmacol Ther 2009;85:86-98.

[5] Steinman MA, Landefeld CS, Rosenthal GE, Berthenthal D, Sen S, Kaboli JP. Polypharmacy and prescribing quality in older people. J Am Geriatr Soc 2006;54:1516-23.

[6] Koh Y, Kutty FBM, Li SC. Drug-related problems in hospitalized patients on polypharmacy: the influence of age and gender. Ther Clin Risk Manag 2005;1:39-48.

[7] Lund BC, Camahan RM, Egge JA, Chrischilles EA, Kaboli PJ. Inappropriate prescribing predicts adverse drug events in older adults. Ann Pharmacother 2010, doi: 10.1345/aph.1M657.

[8] Kaufman DW, Kelly JP, Rosemberg L, Anderson TE, Mitchell AA. Recent patterns of medication use in the ambulatory adult population of the United States: the Slone survey. JAMA 2002;287:337-44.

[9] Corsonello A, Pedone C, Corica F, Antonelli Incalzi R, on behalf of the Gruppo Italiano di Farmacovigilanza (GIFA) investigators. Polypharmacy in the elderly patients at discharge from the acute care hospital. Ther Clin Risk Manage 2007;3:197-203.

[10] Smith S, O'Dowd T. Chronic diseases: what happens when they come in multiples? Br J Gen Pract 2007:57:268-70.

[11] Mercer SW, Smith SM, Wyke S, O'Dowd T, Watt GC. Multimorbidity in primary care: developing the research agenda. Fam Pract 2009;26:79-80.

[12] de Groot V, Beckerman H, Lankhorst GJ, Bouter LM. How to measure comorbidity. a critical review of available methods. J Clin Epidemiol 2003 Mar;56:221-9.

[13] Vogeli C, Shields AE, Lee TA, Gibson TB, Marder WD, Weiss KB, et al. Multiple chronic conditions: prevalence, health consequences, and implications for quality, care management and costs. J Gen Intern Med 2007;22:391-5. 
[14] Marengoni AB, Winblad B, Karp A, Fratiglioni L. Prevalence of chronic diseases and multimorbidity among the elderly population in Sweden. Am J Public Health 2008;98:1198-200.

[15] Marengoni A, Rizzuto D, Wang HX, Winblad B, Fratiglioni L. Patterns of chronic multimorbidity in the elderly population. J Am Geriatr Soc 2009;57:225-30.

[16] Tooth L, Hockey R, Byles J, Dobson A. Weighted multimorbidity indexes predicted mortality, health service use, and health-related quality of life in older women. J Clin Epidemiol 2008:61:151-9.

[17] Wehling M. Multimorbidity and polypharmacy: how to reduce the harmful drug load and yet add needed drugs in the elderly? Proposal of a new drug classification: fit for the aged. J Am Geriatr Soc 2009;57:560-1.

[18] Volpe M, Chin D, Paneni F. The challenge of polypharmacy in cardiovascular medicine. Fundam Clin Pharmacol Feb 2010;24:9-17 Epub 2009 Oct 9.

[19] Good CB. Polypharmacy in elderly patients with diabetes. Diabetes Spectr 2002;15:240-48.

[20] Boyd CM, Ritchie CS, Tipton EF, Studenski SA, Wieland D. From bedside to bench: summary from the American Geriatrics Society/National Institute on Aging Research Conference on comorbidity and multiple morbidity in older adults. Aging Clin Exp Res 2008;20:181-8.

[21] Marengoni A, Bonometti F, Nobili A, Tettamanti M, Salerno F, Corrao S, et al. Inhospital death and adverse clinical events in elderly patients according to disease clustering: the REPOSI study. Rejuvenation Res 2010;13:469-77.

[22] Charlson ME, Pompei P, Ales KL, MacKenzie CR. A new method of classifying prognostic comorbidity in longitudinal studies: development and validation. J Chronic Dis 1987;40:373-83.
[23] Simonson W, Feinberg JL. Medication-related problems in the elderly. Defining the issues and identifying solutions. Drug Aging 2005;22:559-69.

[24] Nobili A, Licata G, Salerno F, Pasina L, Tettamanti M, Franchi C, et al. Polypharmacy, length of hospital stay, and in-hospital mortality among elderly patients in internal medicine wards. The REPOSI study. Eur J Clin Pharmacol 2011;67: 507-19.

[25] Bernardini B, Meinecke C, Zaccarini C, Bongiorni N, Fabbrini S, Gilardi C, et al. Adverse clinical events in dependent long-term nursing home residents. J Am Geriatr Soc 1993;41:105-11.

[26] Grant RW, Devita NG, Singer DE. Polypharmacy and medication adherence in patients with type 2 diabetes. Diabetes Care 2003;26:1408-12.

[27] Boyd CM, Darer J, Boult C, Fried LP, Boult L, Wu AW. Clinical practice guidelines and quality of care for older patients with multiple comorbid diseases: implications for pay for performance. JAMA 2005;294:716-24.

[28] Tinetti ME, Bogardus ST, Agostini JV. Potential pitfalls of disease-specific guidelines for patients with multiple conditions. N Engl J Med 2004;351:2870-4.

[29] Grimmsmann T, Himmel W. Polypharmacy in primary care practices: an analysis using a large health insurance database. Pharmacoepidemiol Drug Saf 2009, doi:10.1002/pds.1841.

[30] Sanoa M, Amatniekb J, Feelyc M, Sinyakd F, Holtonb D, Aschere S, et al. Undertreatment of patients with Alzheimer's disease in an elderly United States population. Alzheimers Dement October 2005;1:136-44. 